Andrew G. Renehan, Sacha Howell, and Anthony Howell, University of Manchester; Michelle Harvie and Anthony Howell, University Hospital of South Manchester NHS Foundation Trust, Manchester; Ramsey I. Cutress, University Hospitals Southampton; Ramsey I. Cutress, University of Southampton, Southampton, United Kingdom; Michael Leitzmann, University of Regensburg, Regensburg; and Tobias Pischon, Max Delbrück Center for Molecular Medicine (MDC), Berlin-Buch, Germany

Published online ahead of print at www.jco.org on November 7, 2016.

Authors' disclosures of potential conflicts of interest are found in the article online at www.jco.org. Author contributions are found at the end of this article.

Corresponding author: Andrew G. Renehan, PhD, FRCS, Department of Surgery, Division of Molecular \& Clinical Cancer Sciences, School of Medical Sciences, Faculty of Biology, Medicine and Health, University of Manchester, Manchester Academic Health Science Centre, The Christie NHS Foundation Trust, Wilmslow Road, Manchester M20 4BX United Kingdom; e-mail: andrew. renehan@ics.manchester.ac.uk.

(C) 2016 by American Society of Clinical Oncology

0732-183X/16/3435w-4284w/\$20.00

DOI: 10.1200/JCO.2016.69.1899

\title{
How to Manage the Obese Patient With Cancer
}

Andrew G. Renehan, Michelle Harvie, Ramsey I. Cutress, Michael Leitzmann, Tobias Pischon, Sacha Howell, and Anthony Howell

\section{$\begin{array}{llllllll}A & B & \mathbf{S} & \mathbf{T} & \mathbf{R} & \mathbf{A} & \mathbf{C} & \mathbf{T}\end{array}$}

\section{Purpose}

Obesity (body mass index $[\mathrm{BMI}] \geq 30 \mathrm{~kg} / \mathrm{m}^{2}$ ) is common among patients with cancer. We reviewed management issues in the obese patient with cancer, focusing on how obesity influences treatment selection (including chemotherapy dosing), affects chemotherapy toxicity and surgical complications, and might be a treatment effect modifier.

\section{Methods}

The majority of evidence is drawn from observational studies and secondary analyses of trial data, typically analyzed in $\mathrm{N} \times 3 \mathrm{BMl}$ categories (normal weight, overweight, and obese) matrix structures. We propose a methodological framework for interpretation focusing on sample size and composition, nonlinearity, and unmeasured confounding

\section{Results}

There is a common perception that obesity is associated with increased treatment-related toxicity. Accordingly, cytotoxic chemotherapy dose reduction is common in patients with elevated BMI. Contrary to this, there is some evidence that full dosing in obese patients does not result in increased toxicity. However, these data are from a limited number of regimens, and fail to fully capture cytotoxic drug pharmacodynamics and pharmacokinetic variability in obese patients. Among patients undergoing surgery, there is evidence that elevated BMI is associated with increased perioperative mortality and increased rates of infectious complications. A novel finding is that these relationships hold after surgery for malignancy, but not for benign indications. There are biologic plausibilities that obesity might be an effect modifier of treatment, but supporting evidence from clinical studies is inconsistent.

\section{Conclusion}

In line with the ASCO 2012 guidelines, chemotherapy dosing is probably best performed using actual body weight in obese patients. However, specific regimens known to be associated with increased toxicity in this group should be used with caution. There is no guidance on dose for obese patients treated with biologic agents. Currently, there are no specific recommendations for the surgical management of the obese patient with cancer.

\section{J Clin Oncol 34:4284-4294. (C) 2016 by American Society of Clinical Oncology}

\section{INTRODUCTION}

Excess body weight, commonly expressed as elevated body mass index (BMI; overweight and obesity), is an established risk factor for several incident adult cancers. ${ }^{1}$ The International Agency for Research on Cancer ${ }^{2}$ lists 13 obesity-related cancers, which include esophageal adenocarcinoma; cancers of the gastric cardia, colorectum, liver, gallbladder, pancreas, postmenopausal breast, endometrium, ovary, kidney (renal cell), and thyroid; meningioma; and multiple myeloma. Thus, for many cancer types, the proportions of patients with obesity (BMI $\geq 30 \mathrm{~kg} / \mathrm{m}^{2}$ ) are high. For example, the ranges ( $11 \%$ to near $50 \%$ ) of the proportions of obese patients by some cancer types are endometrial, $39 \%$ to $47 \%$; renal cell carcinoma, $42 \%$; rectal, $18 \%$ to $37 \%$; esophageal adenocarcinoma, 35\%; GI and colon, $18 \%$ to $26 \%$; ovarian, $12 \%$ to $15 \%$; breast, $11 \%$ to $27 \%$; and metastatic colorectal cancer, 12\% (Data Supplement).

We review management issues in the obese patient with cancer, focusing on how obesity influences treatment selection (including chemotherapy dosing), affects chemotherapy toxicity and surgical complications, and might be a treatment effect modifier. We also include a number of specific miscellaneous aspects that are relevant to the 
treatment of the obese patient with cancer, but are not covered elsewhere in this Special Issue.

\section{METHODS}

The majority of evidence is drawn from observational studies and secondary analyses of trial data, typically analyzed in $\mathrm{N} \times 3$ BMI categories (normal weight, overweight, and obese) matrix structures. We first propose a methodological framework to interpret this structure. Because of the wide scope of this review, a formal literature search of observational studies was deemed impracticable.

\section{Sample Size and Composition}

Case studies in the Data Supplement illustrate the following: small study size can result in both type 1 and type 2 statistical errors in relationships of proportions of interest (eg, complications) and BMI categories (for this reason, in this review, we have cited studies with greater than 1,000 participants, wherever possible); tabulations with greater than two rows of interest (eg, three histologic grades) can be difficult to interpret; and for the same effect size between obese versus normal-weight individuals, with an increasing size of the proportions of obese within a cohort, there is an increased likelihood of statistical significance.

\section{Obesity and Outcomes As Nonlinear Relationships}

Obesity is associated with increased risk of incident cancers ${ }^{3}$ and may also be associated with poorer prognosis, especially in breast cancer (and dealt with elsewhere in this Special Issue ${ }^{4}$ ). These relationships might be nonlinear. Similarly, there might be several types of nonlinear relationships between BMI and the event of interest closer to the treatment window, such as perioperative mortality. These are illustrated in Fig 1 and might include U-shaped curve; inverse J-shaped curve, as observed between BMI and 30-day mortality by Mullen et $\mathrm{al}^{5}$ in their analysis of 2,258 patients who underwent major intraabdominal cancer surgery; a bimodal curve, as observed between BMI and in-hospital mortality after coronary artery bypass graft $(\mathrm{CABG})$ surgery ${ }^{6}$; and a fourth relationship known as the obesity paradox. ${ }^{7}$ The latter is the observation of an unexpected risk reduction in an outcome of interest (usually mortality, but could be peritreatment mortality) among individuals with elevated BMI $\left(\geq 25 \mathrm{~kg} / \mathrm{m}^{2}\right)$, in whom an increased risk is anticipated, compared with those of normal weight $\left(18.5\right.$ to $\left.25 \mathrm{~kg} / \mathrm{m}^{2}\right)$.

Once the many types of nonlinear relationships are recognized, one can appreciate that simple BMI categorizations into normal weight, overweight, and obese might be misleading. For example, in scenario (C) in Figure 1 , a patient with a BMI $=30 \mathrm{~kg} / \mathrm{m}^{2}$ might have a similarly low probability of a complication as a normal-weight patient, but this might hide the possibility that this complication is substantially increased in higher obese states.

\section{Unmeasured Confounding}

For cancer incidence (cohort) studies, there is usually a large array of variables (potential confounders), including smoking status, alcohol consumption, level of education, and socioeconomic status. Taking account of these potential confounders and effect modifiers is important. For example, stratification by smoking status is relevant in the interpretation of
A

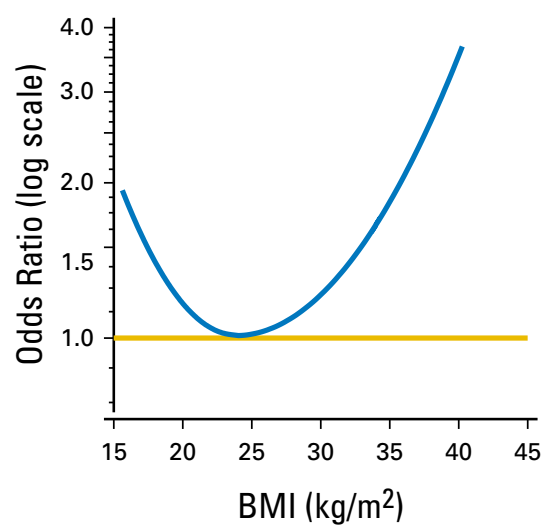

C

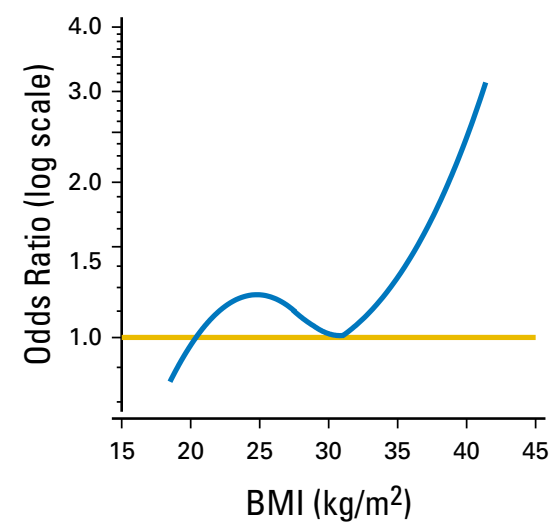

\section{B}

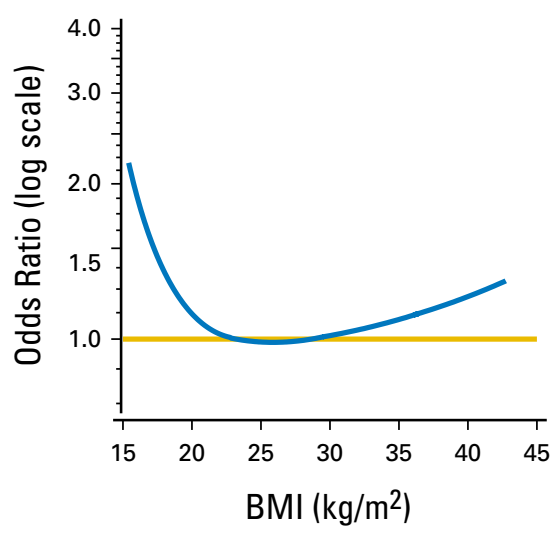

D

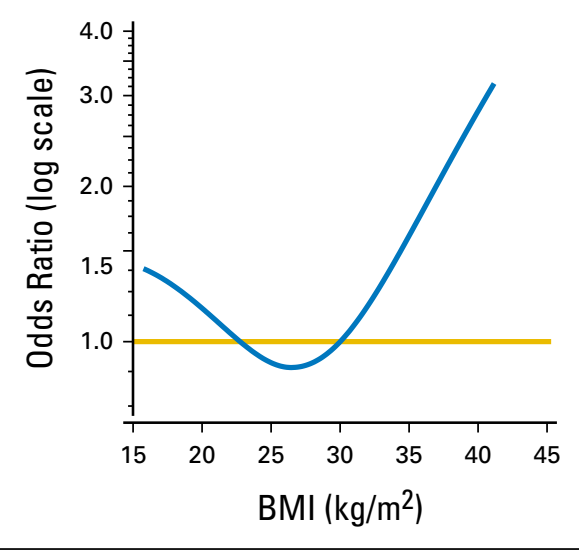

Fig 1. Schematic diagrams of various nonlinear patterns of relationships between body mass index (BMI) and odds ratio of outcome of interest (for example, perioperative mortality). (A) Ushaped; (B) inverse J-shaped; (C) bimodal curve; and (D) obesity paradox (the latter modified from Lennon et $\mathrm{al}^{7}$ ). 
studies of obesity exposure and incident cancer risk, ${ }^{8}$ because smokers have lower mean BMI values compared with those of never smokers. Unfortunately, data on smoking status are infrequently collected in oncology trials (ie, an unmeasured confounder), yet smoking might have major influence on early outcomes such as postoperative complications, peritreatment mortality, and toxicity.

\section{RESULTS}

\section{Treatment Selection}

Selection for specific treatment modalities. Studies have addressed the influence of obesity on the decision to offer certain types of cancer treatments, in specific settings. For example, a review of the National Comprehensive Cancer Network centers' breast cancer data ( $\mathrm{N}=9,527$; stages I, II, and III; 1997 to 2007) showed that BMI had no effect on the decision to offer adjuvant chemotherapy. ${ }^{9}$ Similarly, in rectal cancer, there appears to be no difference in the decision to perform an abdomino-perineal resection versus an anterior resection (a colostomy avoiding operation) between obese versus nonobese patients. ${ }^{10}$

In contrast, obesity negatively influences the decision for immediate breast reconstruction after mastectomy. ${ }^{11}$ In 2007, van de Poll-Franse et al, ${ }^{12}$ using broad modalities of treatments (surgery, radiotherapy, and chemotherapy) recorded in the Eindhoven Cancer Registry, concluded that patients with diabetes (an obesity-related condition) and cancer of the esophagus, colon, breast, or ovary were treated less aggressively (received fewer treatment modalities) compared with those without diabetes. However, this simplistic classification of aggressiveness of treatment is probably not applicable today because present-day management is both multimodal and across multiple phases.

Chemotherapy dosing. In contrast, once a decision is made to offer chemotherapy, there is a large volume of data showing that oncologists frequently reduce doses when prescribing in obese patients because of concerns about toxicity. This philosophy has been challenged for a long time. ${ }^{13}$ Table 1 summarizes a number of studies evaluating chemotherapy dose reductions according to BMI categories in breast, ${ }^{14,15}$ colorectal, ${ }^{16,17}$ and ovarian ${ }^{18,19}$ cancers. This tabulation illustrates that, even within trials, obesity is associated frequently with reduced chemotherapy intensity (ie, reduced frequency and/or failure to complete the prescribed regimen). For example, in an ovarian cancer trial, $66 \%$ of obese patients received suboptimal chemotherapy intensity compared with $30 \%$ for those of normal weight. ${ }^{18}$

What are the consequences of the fact that a high proportion of obese patients with cancer are treated by reduced doses? Among trials where participants received reduced doses of adjuvant chemotherapy, and where the adjuvant chemotherapy is known to improve survival, there is evidence in several cancer types (breast, ${ }^{20-22}$ colorectal, ${ }^{16,23}$ lung, ${ }^{24}$ and lymphoma ${ }^{21}$ ) that survival advantages are lost and outcomes are similar to those of the untreated or control cohorts. Importantly, the implication of this is that the observed adverse prognosis associated with obesity in many cancer types (discussed in other reviews in the Special Issue ${ }^{4}$ ) may reflect confounding as a result of suboptimal chemotherapy dosing and reduced therapeutic effect relative to normal-weight patients with cancer, ${ }^{25}$ rather than obesity per se.

\section{Impact of Obesity on Treatment Complications}

Chemotherapy toxicity. Hourdequin et $\mathrm{al}^{26}$ recently reviewed and meta-analyzed data that evaluated toxic effects of chemotherapy dosing using actual body weight (ABW) in obese versus normal-weight patients with cancer. Against conventional expectations, the summary risk estimates initially suggested that obese patients experience less hematologic toxicity when dosed using $\mathrm{ABW}$, and that among patients in trials where full chemotherapy doses were received, any grade 3/4 toxicities were fewer in obese versus normal-weight participants (summary odds ratio favoring obese patients, $0.75 ; 95 \% \mathrm{CI}, 0.65$ to 0.87 ). However, such conclusions rely heavily on colorectal trials investigating fluorouracil-based regimens, and other trials in breast cancer and lymphoma showed no effect. ${ }^{26}$ More recent data from 3,023 patients with breast cancer, published after this metaanalysis, demonstrate an increase in severe toxicity in ABWdosed obese patients given dose-intense anthracycline- and taxane-containing regimens. ${ }^{27}$

A further dimension is that BMI is a crude approximation of body adiposity and fails to fully capture cytotoxic drug pharmacodynamic and pharmacokinetic variability in obese patients. In the largest analysis to date $(\mathrm{N}=1,206)$ of the effect of obesity on the pharmacokinetics of cytotoxic drugs, Sparreboom et $\mathrm{al}^{28}$ showed drug-specific interactions between BMI and pharmacokinetic clearance, and sex-specific interactions for drugs such as doxorubicin.

There is a paucity of toxicity data on new biologic agents in obese patients with cancer. One recent review demonstrated increased cardiac toxicity associated with Herceptin administration in obese patients. ${ }^{29}$ Traditionally, Herceptin has been dosed per kilogram when an intravenous route is used. However, in many countries (but not in the United States), the administration has been changed to subcutaneous delivery as a single standard 600-mg dose. Detailed pharmacokinetic data show that this alteration results in reduced exposure in obese patients, but the impact on toxicity and survival has yet to be assessed. ${ }^{30}$

In conclusion, data in large adjuvant trial databases should be analyzed to increase our understanding. The observation that fat distribution may have a greater effect on cytotoxic clearance than obesity per se, as well as the complexities of the influence of genetics on pharmacokinetics, suggests that detailed prospective studies of tailoring chemotherapy doses to the individual are required. ${ }^{31,32}$

Surgical complications and perioperative mortality. There is a common perception that obesity is associated with increased postsurgical morbidity and mortality, but there are caveats to this dictum. Table 2 lists studies evaluating relationships among elevated BMI, major complications, and perioperative mortality after nonbariatric, nonvascular general surgery. Many studies were cancer only ${ }^{5,33-36}$ whereas others included benign and malignant indications, ${ }^{37-39}$ which were included to demonstrate that there might be an effect modification by indication. The summary from this tabulation is that elevated BMI is associated with increased perioperative mortality and increased rates of infectious complications, but that there are inconsistent associations between elevated BMI categories and composite (eg, Clavien-Dindo III/IV) or total postsurgical complications. Relationships might be site specific. Thus, for example, for breast reconstruction after 


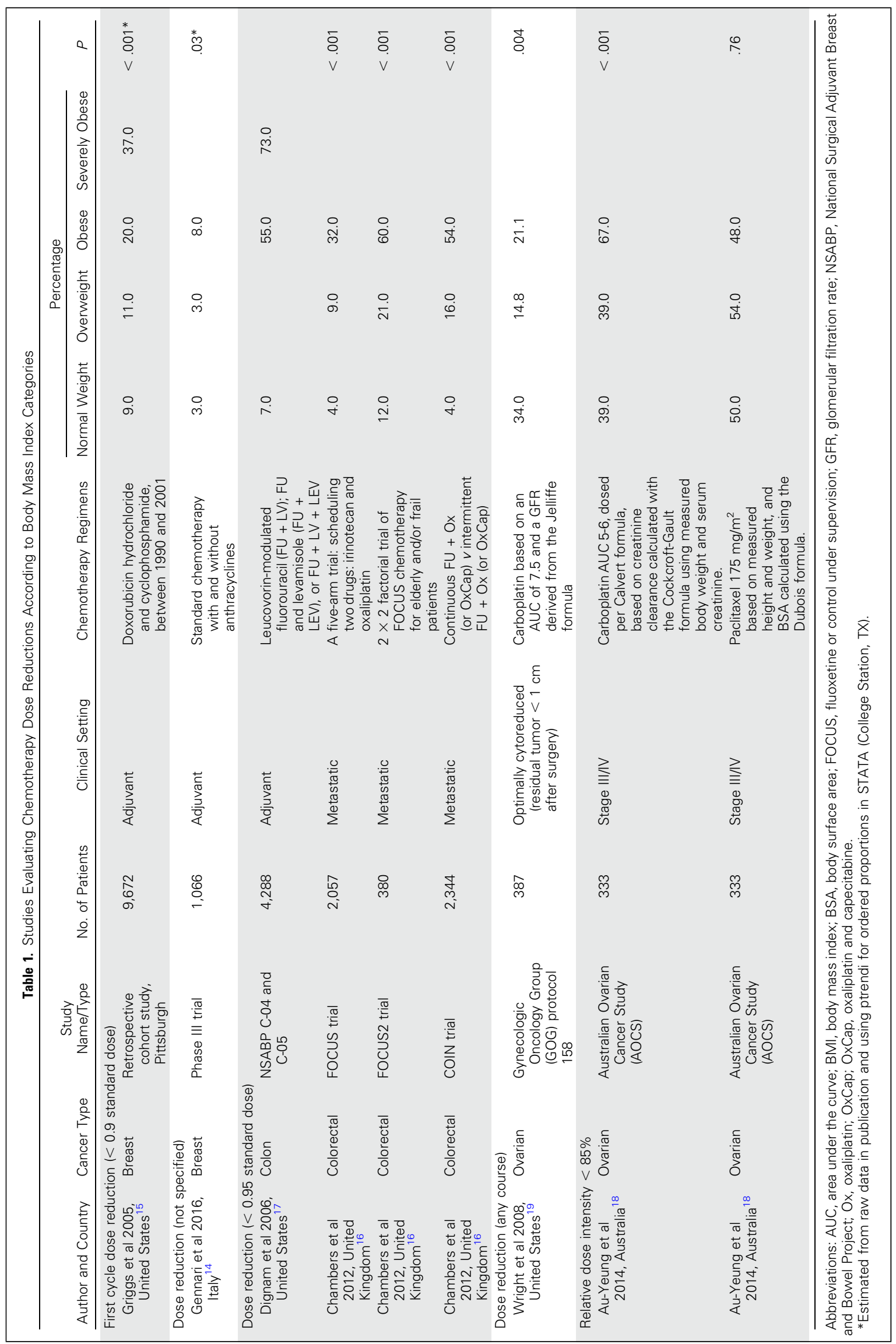




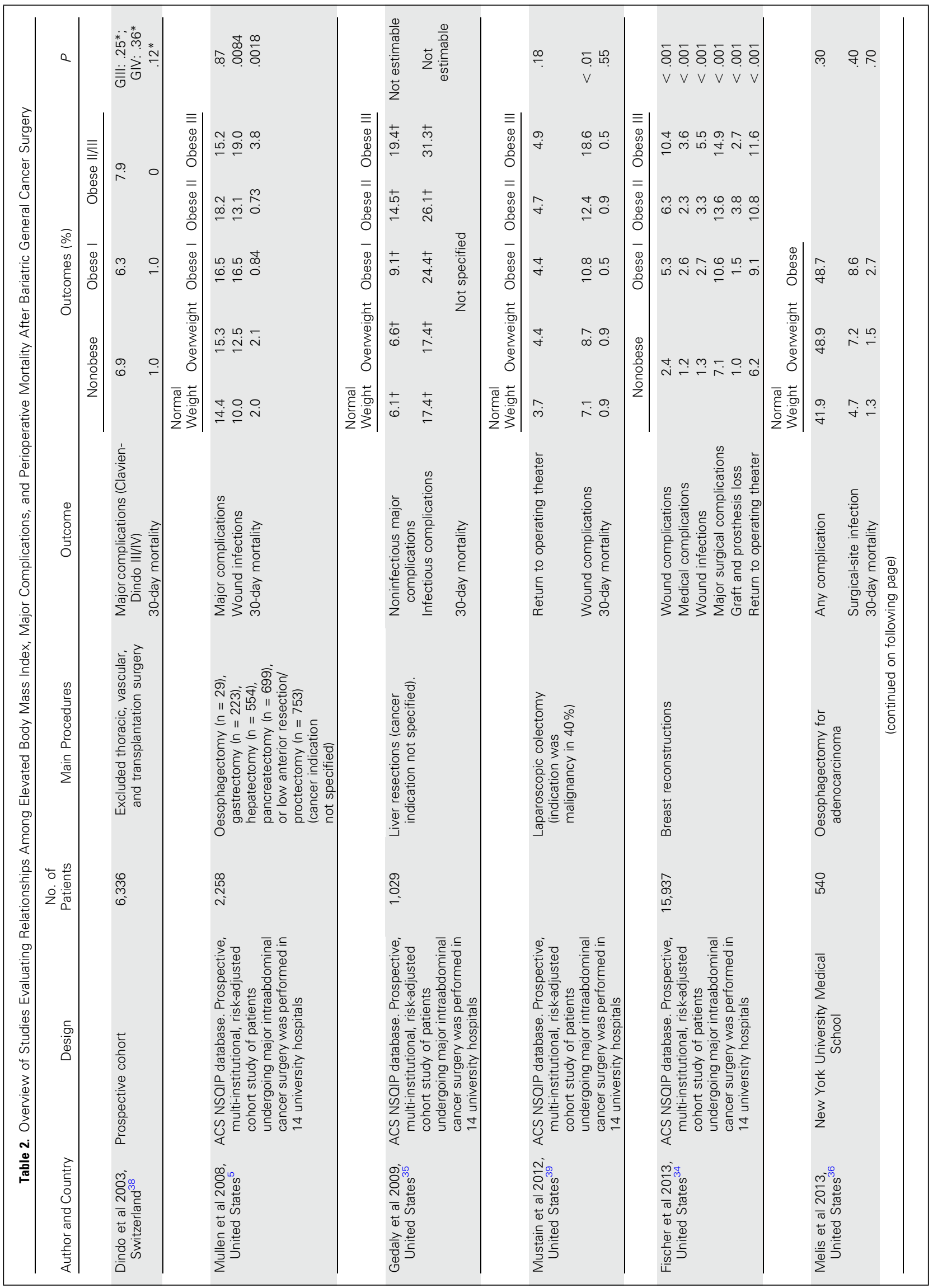




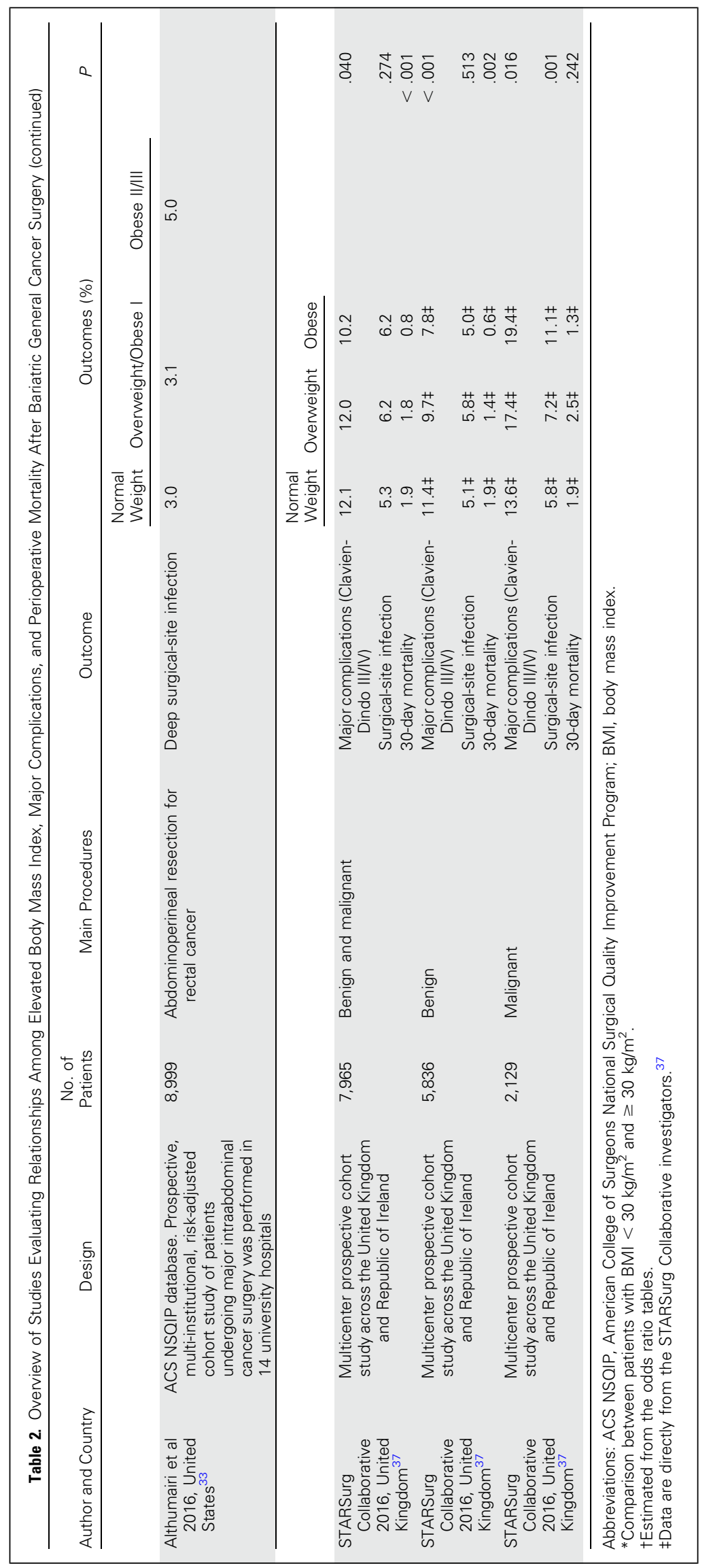


mastectomy, obesity is likely to be an adverse factor for wound and infectious complications. ${ }^{34,40}$

What is interesting is the emerging evidence that the indication for surgery might be an effect modifier. This is clearly illustrated in the recently published STARSurgUK study, ${ }^{37}$ where there were no effects of increased BMI on complications after surgery for benign disease (indeed, there was some evidence of an inverse relationship), but clear (positive) relationships between BMI and postsurgical complications in cases where the surgical indication was malignant disease. This is an important new observation that may explain previous inconsistencies in the literature. It could also reflect that surgeons are highly selective or even avoid operating on obese individuals for benign conditions (and even advocate weight loss before some surgeries for benign indications).

There are other observations worth mentioning. For patients undergoing abdominal laparoscopic surgery for intraabdominal malignancy, there is a recognized increased risk for open conversion with increasing BMI. For the Cleveland Clinic Colorectal Laparoscopic Conversion Score, ${ }^{41} \mathrm{BMI}$ is a key predictor; eg, for a team with 75 to $100-$ patient experience, the predicted conversion rate increases from $2.4 \%$ in subjects with a BMI $<22 \mathrm{~kg} / \mathrm{m}^{2}$ to $7.4 \%$ in subjects with a BMI $>28.5 \mathrm{~kg} / \mathrm{m}^{2}$ undergoing right hemicolectomy surgery. Obesity might have an impact on intermediate-term complications after abdominal cancer surgery, such as incisional and parastomal hernia development. The US Muscle and Adiposity Research Consortium $^{42}$ reported a rate of $21 \%$ incisional hernia formation (median time, 12 months), and that visceral obesity (quantified on routine computed tomography imaging) was a better predictor than BMI for this occurrence.

\section{Obesity As a Treatment Effect Modifier}

It is increasingly recognized that the response to specific cancer therapies differs significantly as a result of different tumor and patient characteristics. These characteristics are termed treatment effect modifiers or treatment predictive biomarkers, and are distinct from prognostic biomarkers. ${ }^{43}$ Obesity is a potential predictive biomarker.

In the setting of aromatase inhibitors (AIs) as hormonal therapy for breast cancer, because obesity is associated with increased peripheral aromatase activity (the target for AIs), it is hypothesized that these agents might be less effective in overweight and obese women (addressed by Goodwin ${ }^{44}$ and within sections of this Special Issue ${ }^{45}$ ). Secondary analyses of four randomized trials found inconsistent results ${ }^{44}$ and concluded that the findings did not support the use of BMI as a treatment predictive biomarker of AIs ( $v$ tamoxifen) in the adjuvant setting in women with postmenopausal breast cancer.

There is some evidence that antiangiogenic agents, such as bevacizumab (dosed per kilogram of weight rather than body surface area), might be less effective in obese patients. Excess adiposity is associated with increased circulating levels of vascular endothelial growth factor, ${ }^{46}$ a key regulator of tumor angiogenesis and the main target for bevacizumab antibody therapy; hence, there is a biologic plausibility that obesity might be a potential predictive biomarker in this setting. This has been tested in the settings of metastatic colorectal cancer $^{47,48}$; metastatic renal cell carcinoma ${ }^{49,50}$; and advanced ovarian cancer ${ }^{51}$ (listed in Table 3 ).

Several of these studies were retrospective, had small sample sizes, lacked controls of conventional treatment arms, and were underpowered to perform tests for interaction; not unexpectedly, the findings are inconsistent. Some studies support the notion that antiangiogenic agents are less effective in obese patients with metastatic colorectal ${ }^{47}$ and advanced ovarian cancer ${ }^{51}$; the opposite is noted in metastatic renal cancer ${ }^{49,50}$; and no difference was seen in the largest analysis, which was a secondary analysis of two trials in metastatic colorectal cancer. ${ }^{48}$ We conclude that currently, obesity is not a treatment predictive biomarker for antiangiogenic therapies in these cancers.

\section{Miscellaneous Matters}

There are a number of issues that require specific discussion regarding the obese patient with cancer, which are not covered elsewhere in this Special Issue.

Weight gain during chemotherapy. All discussion on this matter relates to women with breast cancer. Historically, women who received first-generation nonanthracycline-containing chemotherapy regimens received concomitant corticosteroid therapy, and this contributed substantially to weight gain. Weight gain continues to be seen in women on contemporary regimens of 4.5 to 6 months of anthracycline and taxane chemotherapy, but again, concomitant administration of corticosteroid may contribute. Examples of prospective studies from the United States, ${ }^{52}$ Europe, ${ }^{53}$ and $\mathrm{Asia}^{54}$ report that $30 \%$ to $50 \%$ of women gain $>5 \%$ of body weight, with a mean weight change of 2 to $3 \mathrm{~kg}$ in the first year after diagnosis, although this is not reported in all cohorts. ${ }^{55-58}$

Greatest weight gain is observed among women who are premenopausal, have a healthy weight at diagnosis, ${ }^{59}$ stop smoking after diagnosis, ${ }^{60}$ or experience a chemotherapy-induced menopause. ${ }^{58,61}$ Weight gains may be greater with cyclophosphamide, methotrexate, and fluorouracil compared with anthracycline $(2.9 \mathrm{~kg}[5 \%] v 0.9 \mathrm{~kg}$ $[1 \%]),{ }^{62}$ but this is not a consistent finding. ${ }^{52,61,63}$ Weight is gained both during and after the chemotherapy period, ${ }^{58,64}$ and has been shown to persist when measured at 3 years ${ }^{52}$ and 6 years ${ }^{65}$ after diagnosis.

What are the consequences of chemotherapy-related weight gain? Playdon et $\mathrm{al}^{66}$ addressed this question in a meta-analysis of 23,832 cancer cases from seven cohorts and two chemotherapy trials. They reported that compared with women who maintained stable weight, those who experienced $\geq 10 \%$ weight gains after diagnosis had increased overall mortality (hazard ratio [HR], 1.23; 95\% CI, 1.09 to 1.39 ), and to a lesser extent increased breast cancer mortality (HR, 1.17; 95\% CI, 1.00 to 1.38 ). But there was no association between weight gain and recurrence. However, the metaanalysis highlighted considerable between-study heterogeneity and on close scrutiny, most of the increased risk was within the first years after cancer diagnosis and driven by two studies, ${ }^{67,68}$ raising concerns of reverse causality.

A recently reported pooled project analysis, from the WHEL, LACE, and NHS cohorts, ${ }^{69}$ addressed the question of specificity of associations among 6,596 women with estrogen receptor-positive tumors, and in contrast to the Playdon review, ${ }^{66}$ linked $\geq 10 \%$ weight gain with increased risk of late recurrence, defined 


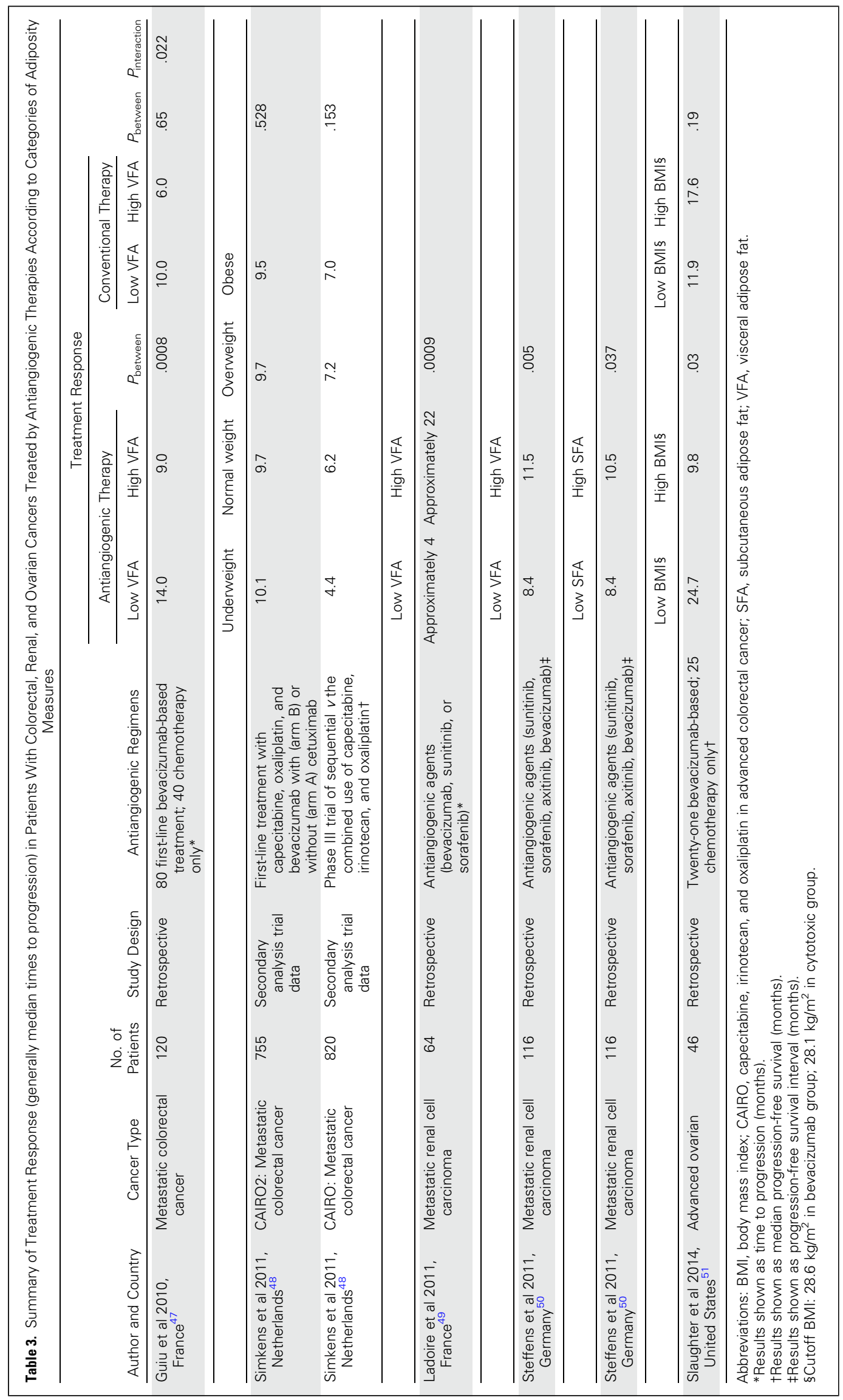


as $>5$ years after diagnosis ( $\mathrm{HR}, 1.24 ; 95 \% \mathrm{CI}, 1.00$ to 1.53 ). However, this significant finding was isolated; no associations were seen for any weight gain and late all-cause mortality. Several randomized trials of lifestyle and weight control interventions in breast cancer survivors, with survival end points, are currently ongoing and covered elsewhere in this special issue. ${ }^{70}$ However, for the most part, recruitment is after treatment and these studies are unlikely to directly address the chemotherapy-related weight gain conundrum.

Excess body weight, noncancer and cardiovascular mortalities. Noncancer deaths may be an issue both during and years after cancer treatment. The ASCO position statement ${ }^{4,71}$ on obesity and cancer recognized that excess body weight might contribute to increased mortality through noncancer deaths, and that of these, approximately half are as a result of cardiovascular disease. However, only a small number of studies have directly addressed this relationship (Data Supplement). For breast cancer, the systematic review and meta-analysis from Chan et $\mathrm{al}^{72}$ identified five studies that evaluated the association between BMI (determined $<12$ months after diagnosis) and noncancer mortality. Compared with normal BMI, the HRs were increased for obesity (but not overweight), but these were not statistically significant. Similarly, the HRs of cardiovascular deaths were increased among obese women compared with those of normal weight (based on pooled data in the Chan meta-analysis ${ }^{72}$ from two studies, where BMI was measured before cancer diagnosis), but these were not statistically significant. In the After Breast Cancer Pooling Project, ${ }^{73}$ which was not included in the Chan meta-analysis, ${ }^{72}$ women who were obese II and III before breast cancer diagnosis (compared with normal weight) were at increased risk of non-breast cancer deaths.

For colorectal cancer, Campbell et $\mathrm{al}^{74}$ demonstrated a significantly increased risk of cardiovascular death with increasing prediagnosis (mean, 7 years) BMI (per $5 \mathrm{~kg} / \mathrm{m}^{2}: \mathrm{HR}, 1.28 ; 95 \% \mathrm{CI}$, 1.04 to 1.58 ), but not postdiagnosis (mean, 1.5 years) BMI (per $5 \mathrm{~kg} / \mathrm{m}^{2}$ : HR, 1.06 ; $95 \% \mathrm{CI}, 0.84$ to 1.33 ). For endometrial cancer, Ward et $\mathrm{al}^{75}$ used the SEER registries, and although they were unable to establish a link with BMI, approximately half of women with endometrial cancer are estimated to be obese. ${ }^{76}$ They concluded that "cardiovascular disease is the leading cause of death among endometrial cancer patients and survivors."

Excess body weight and quality of life. There is a common perception that obesity among cancer survivors has negative consequences on quality of life (QoL). ${ }^{59}$ The prevailing argument is that trials among cancer survivors generally demonstrate that lifestyle interventions that lead to weight reduction are associated with increased physical activity, improved QoL, and less fatigue. ${ }^{71}$ However, these trials are short term and it remains unclear whether a long-term state of excess body weight per se is associated with reduced QoL in cancer survivors. A number of reviews have addressed this question: one study in multiple cancer types ${ }^{77}$; two studies in breast cancer survivors ${ }^{78,79}$; one meta-analysis of 10 studies in colorectal cancer ${ }^{80}$; one meta-analysis of five studies in prostate cancer ${ }^{81}$; one meta-analysis of four studies in endometrial cancer ${ }^{82}$; and one study in ovarian cancer survivors ${ }^{83}$ (Data Supplement).

QoL measures were determined using a variety of different assessment tools and at varying times during survivorship, making direct comparisons difficult. Overall, there were consistent findings that obesity is associated with reduced physical function and reduced vitality, but there was no consistent association for mental health or cognitive function. In a recent review, Smits et $\mathrm{al}^{82}$ recognized that sociodemographic factors and the presence of comorbidities are key determinants of QoL, but these are infrequently available in adjusted models in the cancer survivorship setting. Furthermore, and importantly, almost no study among cancer survivors included appropriately matched noncancer control subjects. One exception is the recently published data from the Long-Term Quality of Life Study in breast cancer survivors, ${ }^{78}$ which reported decreased physical function on SF-36 surveys among obese survivors, but this was also observed among obese individuals without cancer.

\section{PRACTICE RECOMMENDATIONS}

\section{ASCO Clinical Practice Guideline}

The question of chemotherapy dosing in the obese patient was the subject of an ASCO 2012 clinical practice guideline. ${ }^{84}$ The Panel $^{84}$ made six clear clinical recommendations regarding these questions, summarized in Table 4 . The recommendations highlighted that ABW should be used for chemotherapy dosing, regardless of obesity status. Our updated review broadly agrees with these guidelines. However, specific regimens known to be associated with increased toxicity in this group should be used with caution, and this should be taken into account in initial dose selection.

As yet, there is no equivalent guidance for biologic agents (for example, Herceptin and bevacizumab). Currently, there are no specific recommendations for the surgical management of the obese patients with cancer.

\section{Future Research}

This review has highlighted that BMI is a crude measure of body adiposity and fails to capture cytotoxic drug pharmacodynamic

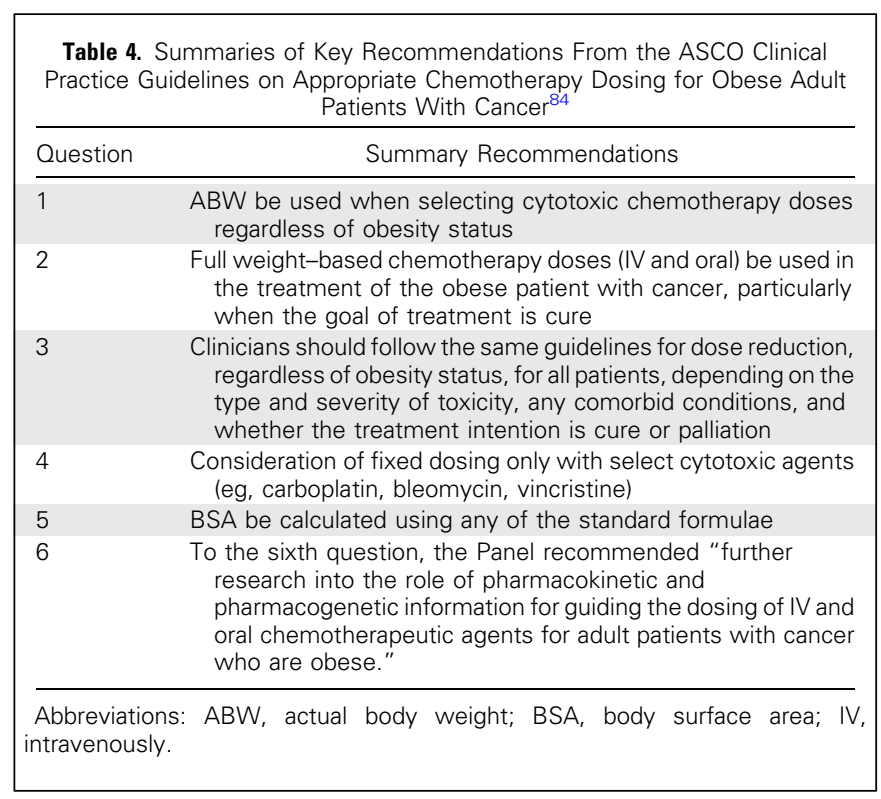


and pharmacokinetic variability in obese patients. There is a need to initiate studies to better assess body composition (for example, computed tomography- or magnetic resonancederived anthropometric measures such as visceral and subcutaneous adipose tissue ${ }^{85}$; and dual-energy $\mathrm{x}$-ray absorptiometry ${ }^{86}$ ) and early outcomes (such as complications and toxicity). Although BMI is the most common measure used to characterize body composition, it cannot distinguish lean mass from fat mass, determine sarcopenic obesity, or characterize body fat distribution. This relationship may differ for individuals with or without cancer, a strategy detailed elsewhere, ${ }^{86}$ and is important to pursue to better define the management of the increasing proportion of obese patients with cancer.

\section{AUTHORS' DISCLOSURES OF POTENTIAL CONFLICTS} OF INTEREST

Disclosures provided by the authors are available with this article at www.jco.org.

\section{AUTHOR CONTRIBUTIONS}

\author{
Conception and design: All authors \\ Data analysis and interpretation: All authors \\ Manuscript writing: All authors \\ Final approval of manuscript: All authors \\ Accountable for all aspects of the work: All authors
}

\section{REFERENCES}

1. Renehan AG, Zwahlen M, Egger M: Adiposity and cancer risk: New mechanistic insights from epidemiology. Nat Rev Cancer 15:484-498, 2015

2. Lauby-Secretan B, Scoccianti C, Loomis D, et al: Body fatness and cancer-Viewpoint of the IARC Working Group. N Engl J Med 375:794-798, 2016

3. Renehan $A G$, Tyson $M$, Egger $M$, et al: Bodymass index and incidence of cancer: A systematic review and meta-analysis of prospective observational studies. Lancet 371:569-578, 2008

4. Ligibel JA, Wollins D: The American Society of Clinical Oncology Obesity Initiative: Rationale, progress, and future directions. Journal of Clinical Oncology Obesity and Cancer Special Issue. In press

5. Mullen JT, Davenport DL, Hutter MM, et al: Impact of body mass index on perioperative outcomes in patients undergoing major intra-abdominal cancer surgery. Ann Surg Oncol 15:2164-2172, 2008

6. Filardo G, Hamilton C, Hamman B, et al: Categorizing BMI may lead to biased results in studies investigating in-hospital mortality after isolated CABG. J Clin Epidemiol 60:1132-1139, 2007

7. Lennon $H$, Sperrin M, Badrick $E$, et al: The obesity paradox in cancer: A review. Curr Oncol Rep 18:56, 2016

8. Song $\mathrm{M}$, Giovannucci E: Estimating the influence of obesity on cancer risk: Stratification by smoking is critical. J Clin Oncol 34:3237-3239, 2016

9. Brewster AM, Etzel C, Zhou R, et al: The impact of obesity on receipt of adjuvant chemotherapy for breast cancer in the National Comprehensive Cancer Network (NCCN) centers. Breast Cancer Res Treat 130:897-904, 2011

10. Bokey L, Chapuis PH, Dent OF: Impact of obesity on complications after resection for rectal cancer. Colorectal Dis 16:896-906, 2014

11. Brennan ME, Spillane AJ: Uptake and predictors of post-mastectomy reconstruction in women with breast malignancy-Systematic review. Eur J Surg Oncol 39:527-541, 2013

12. van de Poll-Franse LV, Houterman S, JanssenHeijnen ML, et al: Less aggressive treatment and worse overall survival in cancer patients with diabetes: A large population based analysis. Int $\mathrm{J}$ Cancer 120:1986-1992, 2007

13. Colleoni M, Li S, Gelber RD, et al: Relation between chemotherapy dose, oestrogen receptor expression, and body-mass index. Lancet 366: 1108-1110, 2005
14. Gennari A, Amadori D, Scarpi $E$, et al: Impact of body mass index (BMI) on the prognosis of highrisk early breast cancer (EBC) patients treated with adjuvant chemotherapy. Breast Cancer Res Treat 159:79-86, 2016

15. Griggs JJ, Sorbero ME, Lyman GH: Undertreatment of obese women receiving breast cancer chemotherapy. Arch Intern Med 165:1267-1273, 2005

16. Chambers $P$, Daniels SH, Thompson LC, et al: Chemotherapy dose reductions in obese patients with colorectal cancer. Ann Oncol 23:748-753, 2012

17. Dignam JJ, Polite BN, Yothers G, et al: Body mass index and outcomes in patients who receive adjuvant chemotherapy for colon cancer. J Natl Cancer Inst 98:1647-1654, 2006

18. Au-Yeung G, Webb PM, DeFazio $A$, et al: Impact of obesity on chemotherapy dosing for women with advanced stage serous ovarian cancer in the Australian Ovarian Cancer Study (AOCS). Gynecol Oncol 133:16-22, 2014

19. Wright JD, Tian C, Mutch DG, et al: Carboplatin dosing in obese women with ovarian cancer: $A$ Gynecologic Oncology Group study. Gynecol Oncol 109:353-358, 2008

20. Bonadonna $G$, Valagussa $P$, Moliterni $A$, et al: Adjuvant cyclophosphamide, methotrexate, and fluorouracil in node-positive breast cancer: The results of 20 years of follow-up. N Engl J Med 332:901-906, 1995

21. Wildiers $H$, Reiser $M$ : Relative dose intensity of chemotherapy and its impact on outcomes in patients with early breast cancer or aggressive lymphoma. Crit Rev Oncol Hematol 77:221-240, 2011

22. Budman DR, Berry DA, Cirrincione CT, et al: Dose and dose intensity as determinants of outcome in the adjuvant treatment of breast cancer. J Natl Cancer Inst 90:1205-1211, 1998

23. Gamelin E, Delva R, Jacob J, et al: Individual fluorouracil dose adjustment based on pharmacokinetic follow-up compared with conventional dosage: Results of a multicenter randomized trial of patients with metastatic colorectal cancer. J Clin Oncol 26: 2099-2105, 2008

24. Arriagada $R$, Le Chevalier $T$, Pignon JP, et al: Initial chemotherapeutic doses and survival in patients with limited small-cell lung cancer. N Engl J Med 329:1848-1852, 1993

25. Hunter RJ, Navo MA, Thaker PH, et al: Dosing chemotherapy in obese patients: Actual versus assigned body surface area (BSA). Cancer Treat Rev 35:69-78, 2009
26. Hourdequin $K C$, Schpero $W L$, McKenna DR, et al: Toxic effect of chemotherapy dosing using actual body weight in obese versus normal-weight patients: A systematic review and meta-analysis. Ann Oncol 24:2952-2962, 2013

27. Furlanetto J, Eiermann W, Marmé $F$, et al: Higher rate of severe toxicities in obese patients receiving dose-dense (dd) chemotherapy according to unadjusted body surface area: Results of the prospectively randomized GAIN study. Ann Oncol mdw315, 2016

28. Sparreboom A, Wolff AC, Mathijssen $\mathrm{RH}$, et al: Evaluation of alternate size descriptors for dose calculation of anticancer drugs in the obese. J Clin Oncol 25:4707-4713, 2007

29. Guenancia C, Lefebvre A, Cardinale, et al: Obesity as a risk factor for anthracyclines and trastuzumab cardiotoxicity in breast cancer: A systematic review and meta-analysis. J Clin Oncol 34:3157-3165, 2016

30. Quartino AL, Hillenbach $C$, Li J, et al: Population pharmacokinetic and exposure-response analysis for trastuzumab administered using a subcutaneous "manual syringe" injection or intravenously in women with HER2positive early breast cancer. Cancer Chemother Pharmacol 77:77-88, 2016

31. Abraham JE, Hiller $L$, Dorling $L$, et al: A nested cohort study of 6,248 early breast cancer patients treated in neoadjuvant and adjuvant chemotherapy trials investigating the prognostic value of chemotherapyrelated toxicities. BMC Med 13:306, 2015

32. Wong $A L$, Seng $K Y$, Ong $E M$, et al: Body fat composition impacts the hematologic toxicities and pharmacokinetics of doxorubicin in Asian breast cancer patients. Breast Cancer Res Treat 144: 143-152, 2014

33. Althumairi AA, Canner JK, Gearhart SL, et al: Risk factors for wound complications after abdominoperineal excision: Analysis of the ACS NSQIP database. Colorectal Dis 18:O260-O266, 2016

34. Fischer JP, Nelson JA, Kovach SJ, et al: Impact of obesity on outcomes in breast reconstruction: Analysis of 15,937 patients from the ACS-NSQIP datasets. J Am Coll Surg 217:656-664, 2013

35. Gedaly R, McHugh PP, Johnston TD, et al: Obesity, diabetes, and smoking are important determinants of resource utilization in liver resection: $A$ multicenter analysis of 1029 patients. Ann Surg 249: 414-419, 2009

36. Melis M, Weber J, Shridhar R, et al: Body mass index and perioperative complications after oesophagectomy for adenocarcinoma: A systematic database review. BMJ Open 3:e001336, 2013 
37. STARSurg Collaborative: Multicentre prospective cohort study of body mass index and postoperative complications following gastrointestinal surgery. Br J Surg 103:1157-1172, 2016

38. Dindo $D$, Muller MK, Weber M, et al: Obesity in general elective surgery. Lancet 361:2032-2035, 2003

39. Mustain WC, Davenport DL, Hourigan JS, et al: Obesity and laparoscopic colectomy: Outcomes from the ACS-NSQIP database. Dis Colon Rectum 55:429-435, 2012

40. Robertson SA, Rusby JE, Cutress RI: Determinants of optimal mastectomy skin flap thickness. Br J Surg 101:899-911, 2014

41. Senagore A, Delaney C, Fazio $V$, et al: Cleveland Clinic Colorectal Laparoscopic Conversion Model. http://www.riskprediction.org.uk/index-ccflap. php

42. Aquina $C T$, Rickles $A S$, Probst $C P$, et al: Visceral obesity, not elevated BMI, is strongly associated with incisional hernia after colorectal surgery. Dis Colon Rectum 58:220-227, 2015

43. Mandrekar SJ, Sargent DJ: Clinical trial designs for predictive biomarker validation: Theoretical considerations and practical challenges. J Clin Oncol 27:4027-4034, 2009

44. Goodwin PJ: Obesity and endocrine therapy: Host factors and breast cancer outcome. Breast 22 (Suppl 2):S44-S47, 2013

45. Jiralerspong S, Goodwin PJ: Obesity and breast cancer prognosis-Evidence, challenges, and opportunities. Journal of Clinical Oncology Obesity and Cancer Special Issue. doi: 10.1200/JCO.2016.68. 4480

46. Renehan AG: Body fatness and bevacizumabbased therapy in metastatic colorectal cancer. Gut 59:289-290, 2010

47. Guiu B, Petit JM, Bonnetain F, et al: Visceral fat area is an independent predictive biomarker of outcome after first-line bevacizumab-based treatment in metastatic colorectal cancer. Gut 59:341-347, 2010

48. Simkens LH, Koopman M, Mol L, et al: Influence of body mass index on outcome in advanced colorectal cancer patients receiving chemotherapy with or without targeted therapy. Eur $\mathrm{J}$ Cancer 47: 2560-2567, 2011

49. Ladoire S, Bonnetain F, Gauthier M, et al: Visceral fat area as a new independent predictive factor of survival in patients with metastatic renal cell carcinoma treated with antiangiogenic agents. Oncologist 16:71-81, 2011

50. Steffens S, Grünwald V, Ringe KI, et al: Does obesity influence the prognosis of metastatic renal cell carcinoma in patients treated with vascular endothelial growth factor-targeted therapy? Oncologist 16:1565-1571, 2011

51. Slaughter KN, Thai $T$, Penaroza $S$, et al: Measurements of adiposity as clinical biomarkers for first-line bevacizumab-based chemotherapy in epithelial ovarian cancer. Gynecol Oncol 133:11-15, 2014

52. Makari-Judson $\mathrm{G}$, Judson $\mathrm{CH}$, Mertens WC Longitudinal patterns of weight gain after breast cancer diagnosis: Observations beyond the first year. Breast J 13:258-265, 2007

53. Lankester KJ, Phillips JE, Lawton PA: Weight gain during adjuvant and neoadjuvant chemotherapy for breast cancer: An audit of 100 women receiving
FEC or CMF chemotherapy. Clin Oncol (R Coll Radiol) 14:64-67, 2002

54. Gu K, Chen X, Zheng $Y$, et al: Weight change patterns among breast cancer survivors: Results from the Shanghai breast cancer survival study. Cancer Causes Control 21:621-629, 2010

55. Chaudhary LN, Wen S, Xiao J, et al: Weight change associated with third-generation adjuvant chemotherapy in breast cancer patients. J Community Support Oncol 12:355-360, 2014

56. Han HS, Lee KW, Kim JH, et al: Weight changes after adjuvant treatment in Korean women with early breast cancer. Breast Cancer Res Treat 114:147-153, 2009

57. Reddy SM, Sadim M, Li J, et al: Clinical and genetic predictors of weight gain in patients diagnosed with breast cancer. Br J Cancer 109 872-881, 2013

58. Freedman RJ, Aziz N, Albanes D, et al: Weight and body composition changes during and after adjuvant chemotherapy in women with breast cancer. J Clin Endocrinol Metab 89:2248-2253, 2004

59. Makari-Judson G, Braun B, Jerry DJ, et al: Weight gain following breast cancer diagnosis: Implication and proposed mechanisms. World J Clin Oncol 5:272-282, 2014

60. Sedjo RL, Byers T, Ganz PA, et al: Weight gain prior to entry into a weight-loss intervention study among overweight and obese breast cancer survivors. J Cancer Surviv 8:410-418, 2014

61. Goodwin PJ, Ennis M, Pritchard KI, et al: Adjuvant treatment and onset of menopause predict weight gain after breast cancer diagnosis. J Clin Oncol 17:120-129, 1999

62. Liu LN, Wen FH, Miaskowski C, et al: Weight change trajectory in women with breast cancer receiving chemotherapy and the effect of different regimens. J Clin Nurs 23:2757-2768, 2014

63. Irwin ML, McTiernan A, Baumgartner $R N$ et al: Changes in body fat and weight after a breast cancer diagnosis: Influence of demographic, prognostic, and lifestyle factors. J Clin Oncol 23:774-782, 2005

64. Harvie MN, Campbell IT, Baildam A, et al: Energy balance in early breast cancer patients receiving adjuvant chemotherapy. Breast Cancer Res Treat 83:201-210, 2004

65. Vagenas D, DiSipio T, Battistutta D, et al: Weight and weight change following breast cancer: Evidence from a prospective, population-based, breast cancer cohort study. BMC Cancer 15:28, 2015

66. Playdon MC, Bracken MB, Sanft TB, et al: Weight gain after breast cancer diagnosis and allcause mortality: Systematic review and meta-analysis. J Natl Cancer Inst 107:djv275, 2015

67. Bradshaw PT, Ibrahim JG, Stevens J, et al: Postdiagnosis change in bodyweight and survival after breast cancer diagnosis. Epidemiology 23 320-327, 2012

68. Nichols HB, Trentham-Dietz A, Egan KM, et al: Body mass index before and after breast cancer diagnosis: Associations with all-cause, breast cancer, and cardiovascular disease mortality. Cancer Epidemiol Biomarkers Prev 18:1403-1409, 2009

69. Nechuta $S$, Chen WY, Cai $H$, et al: A pooled analysis of post-diagnosis lifestyle factors in association with late estrogen-receptor-positive breast cancer prognosis. Int J Cancer 138:2088-2097, 2016
70. Chlebowski RT, Reeves MM: Weight loss randomized intervention trials in women cancer survivors. Journal of Clinical Oncology Special Issue: Obesity and Cancer. In press

71. Ligibel JA, Alfano CM, Courneya KS, et al: American Society of Clinical Oncology position statement on obesity and cancer. J Clin Oncol 32: 3568-3574, 2014

72. Chan DS, Vieira AR, Aune D, et al: Body mass index and survival in women with breast cancer -Systematic literature review and meta-analysis of 82 follow-up studies. Ann Oncol 25:1901-1914, 2014

73. Kwan ML, Chen WY, Kroenke $\mathrm{CH}$, et al: Prediagnosis body mass index and survival after breast cancer in the After Breast Cancer Pooling Project. Breast Cancer Res Treat 132:729-739, 2012

74. Campbell PT, Newton CC, Dehal AN, et al: Impact of body mass index on survival after colorectal cancer diagnosis: The Cancer Prevention Study-II Nutrition Cohort. J Clin Oncol 30:42-52, 2012

75. Ward KK, Shah NR, Saenz CC, et al: Cardiovascular disease is the leading cause of death among endometrial cancer patients. Gynecol Oncol 126: 176-179, 2012

76. Crosbie EJ, Roberts C, Qian W, et al: Body mass index does not influence post-treatment survival in early stage endometrial cancer: Results from the MRC ASTEC trial. Eur J Cancer 48: 853-864, 2012

77. Blanchard CM, Stein K, Courneya KS: Body mass index, physical activity, and health-related quality of life in cancer survivors. Med Sci Sports Exerc 42:665-671, 2010

78. Connor $A E$, Baumgartner $R N$, Pinkston $C M$, et al: Obesity, ethnicity, and quality of life among breast cancer survivors and women without breast cancer: The long-term quality of life follow-up study. Cancer Causes Control 27:115-124, 2016

79. Paxton RJ, Phillips KL, Jones LA, et al: Associations among physical activity, body mass index, and health-related quality of life by race/ethnicity in a diverse sample of breast cancer survivors. Cancer 118:4024-4031, 2012

80. Jansen $L$, Koch $L$, Brenner $H$, et al: Quality of life among long-term ( $\geq 5$ years) colorectal cancer survivors-Systematic review. Eur J Cancer 46: 2879-2888, 2010

81. Allott EH, Masko EM, Freedland SJ: Obesity and prostate cancer: Weighing the evidence. Eur Urol 63:800-809, 2013

82. Smits A, Lopes A, Bekkers R, et al: Body mass index and the quality of life of endometrial cancer survivors-A systematic review and meta-analysis. Gynecol Oncol 137:180-187, 2015

83. Smits A, Lopes A, Das N, et al: Quality of life in ovarian cancer survivors: The influence of obesity. Int J Gynecol Cancer 25:616-621, 2015

84. Griggs JJ, Mangu PB, Anderson H, et al: Appropriate chemotherapy dosing for obese adult patients with cancer: American Society of Clinical Oncology clinical practice guideline. J Clin Oncol 30: 1553-1561, 2012

85. Coe PO, O'Reilly DA, Renehan AG: Excess adiposity and gastrointestinal cancer. Br J Surg 101: 1518-1531, discussion 1531, 2014

86. James FR, Wootton $S$, Jackson A, et al: Obesity in breast cancer-What is the risk factor? Eur J Cancer 51:705-720, 2015 


\section{AUTHORS' DISCLOSURES OF POTENTIAL CONFLICTS OF INTEREST}

How to Manage the Obese Patient With Cancer

The following represents disclosure information provided by authors of this manuscript. All relationships are considered compensated. Relationships are self-held unless noted. I = Immediate Family Member, Inst = My Institution. Relationships may not relate to the subject matter of this manuscript. For more information about ASCO's conflict of interest policy, please refer to www.asco.org/rwc or jco.ascopubs.org/site/ifc.

\section{Andrew G. Renehan}

Honoraria: Janssen-Cilag, Merck Serono

Research Funding: Novo Nordisk

\section{Michelle Harvie}

No relationship to disclose

\section{Ramsey I. Cutress}

Other Relationship: A body composition analyzer (SECA mBCA 515) was provided to the National Institute for Health Research (NIHR)

Southampton Biomedical Research Centre in Nutrition by SECA more than 2 years ago.

\section{Michael Leitzmann}

No relationship to disclose

Tobias Pischon

No relationship to disclose

Sacha Howell

Consulting or Advisory Role: Novartis

Research Funding: Evgen Pharma Ltd

Travel, Accommodations, Expenses: Amgen

Anthony Howell

No relationship to disclose 


\section{Acknowledgment}

We thank Hannah Lennon, University of Manchester, for her contribution to the drawing of the schematic plots in Fig 1. We thank the lead investigators of the STARSurg Collaborative for additional analyses of their published work, ${ }^{37}$ contributing more detail to Table 2 . 